\title{
Application of accretive operators theory to evolutive combined conduction, convection and radiation
}

\section{Maria Michaela Porzio and Óscar López-Pouso}

\begin{abstract}
The accretive operators theory is employed for proving an existence theorem for the evolutive energy equations involving simultaneously conduction, stationary convection (in the sense that the velocity field is assumed to be time independent), and radiation. In doing that we need to use new existence results for elliptic linear problems with mixed boundary conditions and irregular data.
\end{abstract}

\section{Introduction}

Radiation heat transfer may have a great importance in processes at high temperatures. In those cases temperatures are affected by variations in the intensity of radiation, which is the solution of a transfer equation like the neutron transport equation, which also depends on the temperature. This means that one has to solve a coupled system for the temperature $(T)$ and the intensity $(i)$. Transient solutions for the conduction-radiation problem, i.e., when both conduction and radiation affect the evolution of temperatures, are necessary to examine heat transfer and thermal stress behavior for many practical applications. This includes "heat transfer in ceramic components for high temperature use, thermal protection coatings, glass forming for manufacturing, tempering of glass windows, glass envelopes for high intensity lamps, porous burners and insulation systems, liquid-drop and liquid-sheet space radiators, ceramic insulation for atmospheric reentry of spacecraft, and some high temperature components in advanced aircraft engines" [19].

2000 Mathematics Subject Classification: 35D05, 45K05, 80A20, 85A25.

Keywords: radiative heat transfer combined with conduction and convection, accretive operators, mixed boundary conditions, irregular data. 
Existence results for the steady conduction-radiation problem have been proved [11], [12], while the evolutive problem including only radiation has been object of several studies [10], [16], [14] and references therein. Regarding the evolutive convection-radiation problem, it has been proved to be solvable when the velocity field is regular, steady and incompressible [14]. The result of existence given in this paper is, to the authors' knowledge, the first one for the evolutive problem including combined conduction, convection, and radiation.

The use of accretive operators theory in $\mathrm{L}^{1}$ spaces makes this work be the natural continuation of Mercier's paper [16]. The inclusion of convection and conduction leads to the difficulty, pointed out in [14], of proving existence and uniqueness in $\mathrm{L}^{1}$ for $T+\mathbf{v} \cdot \nabla T-\Delta T=\hat{T}$, where $\hat{T}$ is given in $\mathrm{L}^{1}$. This is an interesting problem by itself. In the case of Dirichlet homogeneous boundary conditions the existence of distributional solutions is proved in [8], [9], [2], [3] and in [4]. In the general case of mixed boundary conditions, the existence of weak solutions was not proved even with regular data (that is $\hat{T} \in \mathrm{L}^{2}(\Omega)$ ). Moreover, for what concerns uniqueness results, in [3] (see also [5]) it is also proved that the solution of the homogeneous Dirichlet problem that the authors have constructed satisfies some further regularity conditions (i.e., it is a renormalized solution) and that there is uniqueness in the set of the renormalized solutions. Unfortunately this is insufficient for us because what we need is uniqueness in $W^{1,1}(\Omega)$. Anyway we have solved this problem in [15] under (also nonhomogeneous) Dirichlet boundary conditions or under suitable boundary mixed conditions (see subsection 4.1.2 below).

The paper is organized as follows. In Section 2 we present the equations, under the name the convection-conduction-radiation problem. Section 3 contains the statement of the main theorem (Theorem 3.1), and Section 4 is devoted to its proof. The paper ends with the conclusions in Section 5, and the proofs of some technical lemmas in Section 6 (Appendix).

\section{The mathematical model}

\subsection{Notations}

- $a$ : absorption coefficient [units: $1 / m$ ].

- $c$ : speed of light in the medium $[\mathrm{m} / \mathrm{s}]$.

- $c_{v}$ : specific heat at constant volume $[\mathrm{J} /(\mathrm{kg} \mathrm{K})]$.

- $d \boldsymbol{\omega}$ : surface measure on the unit sphere $S^{2}$.

- $F$ : internal heat source $\left[W / m^{3}\right]$. 
- $i$ : intensity of radiation $\left[J /\left(m^{2} s r\right)\right]$ (here $s r$ stands for steradian, the unit of solid angle).

- $i_{b}$ : intensity of radiation emitted from a blackbody $\left[J /\left(m^{2} s r\right)\right]$. The function $i_{b}$ obeys Planck's law:

$$
i_{b}(\nu, T)=\frac{2 h n^{2} \nu^{3}}{c_{0}^{2}\left(\mathrm{e}^{h \nu / k T}-1\right)}
$$

for a frequency $\nu$ and a temperature $T$, where $h=6.6262 \times 10^{-34}$ $[J \cdot s]$ is Planck's constant, $k=1.3806 \times 10^{-23}[\mathrm{~J} / \mathrm{K}]$ is Boltzmann's constant, $n$ is the refractive index of the medium and $c_{0}=2.9979 \times 10^{8}$ $[\mathrm{m} / \mathrm{s}]$ is the speed of light in the vacuum.

- $\bar{k}$ : thermal conductivity $[W /(m K)]$.

- $\mathbf{q}_{\mathbf{c}}$ : conductive flux vector $\left[W / m^{2}\right]$.

- $\mathbf{q}_{\mathbf{r}}$ : radiant flux vector $\left[W / m^{2}\right]$.

- $T$ : temperature $[K]$.

- v: velocity field $[\mathrm{m} / \mathrm{s}]$.

- $\rho$ : density $\left[\mathrm{kg} / \mathrm{m}^{3}\right]$.

- $\sigma_{s}$ : scattering coefficient $[1 / m]$.

- $\phi$ : scattering phase function [dimensionless]. This function must satisfy the equality

$$
\int_{S^{2}} \phi\left(\boldsymbol{\omega}^{\star}, \boldsymbol{\omega}\right) d \boldsymbol{\omega}=4 \pi \quad \forall \boldsymbol{\omega}^{\star} \in S^{2}
$$

\subsection{Mathematical model}

Let us consider a bounded, open and connected set $\Omega \subset \mathbb{R}^{3}$ with regular $\mathrm{C}^{\infty}$ boundary $\Gamma$, and a bounded time interval $(0, \tau)$. Let us call $Q=\Omega \times(0, \tau)$ and $\Sigma=(0, \infty) \times \Omega \times S^{2} \times(0, \tau)$. For the sake of brevity, we will use the notation $i(\boldsymbol{\omega})=i(\nu, \mathbf{x}, \boldsymbol{\omega}, t)$.

A gas is called participating when it scatters, absorbs, and emits thermal radiation. The following system models the thermal evolution of a participating incompressible gas with given velocity field $\mathbf{v}$ [6], [17], which is being heated by an internal source $F(\mathbf{x}, t)$ :

$$
\begin{array}{r}
\rho c_{v}\left(\frac{\partial T}{\partial t}+\mathbf{v} \cdot \nabla_{\mathbf{x}} T\right)+\nabla_{\mathbf{x}} \cdot\left(\mathbf{q}_{\mathbf{r}}+\mathbf{q}_{\mathbf{c}}\right)=F \text { in } Q . \\
\frac{1}{c} \frac{\partial i}{\partial t}+\boldsymbol{\omega} \cdot \nabla_{\mathbf{x}} i+a\left(i-i_{b}\right)=L(i) \text { in } \Sigma,
\end{array}
$$

where

$$
L(i)=\frac{\sigma_{s}}{4 \pi} \int_{S^{2}}\left[\phi\left(\boldsymbol{\omega}^{\star}, \boldsymbol{\omega}\right) i\left(\boldsymbol{\omega}^{\star}\right)-\phi\left(\boldsymbol{\omega}, \boldsymbol{\omega}^{\star}\right) i(\boldsymbol{\omega})\right] d \boldsymbol{\omega}^{\star} .
$$


The conductive flux vector is given by Fourier's law:

$$
\mathbf{q}_{\mathbf{c}}=-\bar{k} \nabla_{\mathbf{x}} T,
$$

and the radiant flux vector is given by

$$
\mathbf{q}_{\mathbf{r}}=\int_{0}^{\infty} \int_{S^{2}} i \boldsymbol{\omega} d \boldsymbol{\omega} d \nu
$$

If the transient term $(1 / c)(\partial i / \partial t)$ is removed, then (2.4) and (2.6) imply, formally,

$$
\nabla_{\mathbf{x}} \cdot \mathbf{q}_{\mathbf{r}}=\int_{0}^{\infty} \int_{S^{2}} a\left(i_{b}-i\right) d \boldsymbol{\omega} d \nu
$$

Thus, we have the following system, which is expected to be a good model in case that $(1 / c)(\partial i / \partial t)$ is negligible (this is really the case in many applications [17], not in astrophysics neither in short pulsar lasers):

$$
\begin{aligned}
\rho c_{v}\left(\frac{\partial T}{\partial t}+\mathbf{v} \cdot \nabla_{\mathbf{x}} T\right)-\bar{k} \Delta_{\mathbf{x}} T & +\int_{0}^{\infty} \int_{S^{2}} a\left(i_{b}-i\right) d \boldsymbol{\omega} d \nu \\
\frac{1}{c} \frac{\partial i}{\partial t}+\boldsymbol{\omega} \cdot \nabla_{\mathbf{x}} i+a\left(i-i_{b}\right) & =L(i) \text { in } \Sigma .
\end{aligned}
$$

The system (2.8)-(2.9) has been proved to be solvable in the purely radiant case $(\mathbf{v} \equiv \mathbf{0}, \bar{k}=0)[16],[13],[14]$, and in the convective-radiative case $(\bar{k}=0)[14]$. Here we assume that conduction is always present together with radiation, but the convective term may appear or not; in other words, $\bar{k}$ is strictly positive but $\mathbf{v}$ may be $\mathbf{0}$. We will call this problem, together with the initial and boundary conditions, the convection-conductionradiation problem.

\subsubsection{Initial Conditions}

We require that

$$
\begin{aligned}
T(\mathbf{x}, 0) & =T_{0}(\mathbf{x}) \text { in } \Omega, \\
i(\nu, \mathbf{x}, \boldsymbol{\omega}, 0) & =i_{0}(\nu, \mathbf{x}, \boldsymbol{\omega}) \text { in }(0, \infty) \times \Omega \times S^{2} .
\end{aligned}
$$

\subsubsection{Boundary Conditions (from now on, b.c.)}

Recall that $\Gamma$ is the boundary of $\Omega$. We use the notations:

- $D_{\text {in }}=\left\{(\mathbf{x}, \boldsymbol{\omega}) \in \Gamma \times S^{2}: \mathbf{n}(\mathbf{x}) \cdot \boldsymbol{\omega}<0\right\}$, where $\mathbf{n}(\mathbf{x})$ is the outward unit normal at $\mathbf{x} \in \Gamma$.

- $\Gamma_{1}$ is a measurable subset of $\Gamma$.

- $\Gamma_{\mathbf{v}}^{-}=\{\mathbf{x} \in \Gamma: \mathbf{n}(\mathbf{x}) \cdot \mathbf{v}(\mathbf{x})<0\}$. 
For $i$ the b.c. is

$$
i_{\mid(0, \infty) \times D_{\text {in }} \times(0, \tau)}(\nu, \mathbf{x}, \boldsymbol{\omega}, t)=\bar{h}(\nu, \mathbf{x}, \boldsymbol{\omega}),
$$

which means that the inflow of radiant intensity is known and independent of $t$.

For $T$ we consider the mixed b.c.

$$
\begin{aligned}
T_{\mid \Gamma_{1} \times(0, \tau)}(\mathbf{x}, t) & =\bar{g}(\mathbf{x}), \\
\left(\nabla_{\mathbf{x}} T \cdot \mathbf{n}\right)_{\mid\left(\Gamma \backslash \Gamma_{1}\right) \times(0, \tau)}(\mathbf{x}, t) & =0,
\end{aligned}
$$

where $\Gamma_{1}$ is such that $\Gamma_{\mathbf{v}}^{-} \subseteq \Gamma_{1}$.

Remark 2.1 Note that the Dirichlet b.c. $T_{\mid \Gamma \times(0, \tau)}=\bar{g}$ is obtained for the choice $\Gamma_{1}=\Gamma$ in the mixed b.c. (2.13)-(2.14). Moreover, if $\mathbf{v} \cdot \mathbf{n} \geq 0$ on $\Gamma$ (in particular if $\mathbf{v} \equiv \mathbf{0}$, which corresponds to the case of a motionless gas), $\Gamma_{1}$ may be an arbitrary measurable subset of $\Gamma$; in that case, the mixed b.c. (2.13)-(2.14) with the choice $\Gamma_{1}=\emptyset$ becomes homogeneous Neumann b.c.

\section{Main result}

The density $\rho$, the specific heat $c_{v}$ and the conductivity $\bar{k}$ are assumed to be constant and positive. The velocity field $\mathbf{v}$ is assumed to be independent of $t$, and with regularity

$$
\mathbf{v} \in\left(\mathrm{W}^{1, \infty}(\Omega)\right)^{3}, \quad \operatorname{div} \mathbf{v}=0 .
$$

We assume also the following dependences: $a=a(\nu, T(\mathbf{x}, t)), \sigma_{s}=\sigma_{s}(\nu, \mathbf{x})$, being the second one different from the first only due to technical reasons. Lastly, let us assume that

$$
F \in \mathrm{L}^{\infty}(\Omega \times[0, \tau]) ;
$$

$\sigma_{s}$ and $\phi$ are measurable, essentially bounded and nonnegative;

$\phi$ is symmetric, i.e., $\phi\left(\boldsymbol{\omega}, \boldsymbol{\omega}^{\star}\right)=\phi\left(\boldsymbol{\omega}^{\star}, \boldsymbol{\omega}\right)$;

$T_{0} \in \mathrm{L}^{1}(\Omega) ; i_{0} \in \mathrm{L}^{1}\left((0, \infty) \times \Omega \times S^{2}\right) ;$

$\bar{h} \in \mathrm{L}^{1}\left((0, \infty) \times D_{\text {in }},|\boldsymbol{\omega} \cdot \mathbf{n}| d \nu d \gamma d \boldsymbol{\omega}\right)$;

with $\bar{g}$ we denote both $\psi_{\mid \Gamma_{1}}$ or $\psi_{\mid \Gamma}$, where $\psi \in \mathcal{D}(\bar{\Omega})$;

and that, for certain real numbers $m$ and $M$ with $0<m<M$,

$$
a_{\mid(0, \infty) \times[m, M]} \text { is continuous }
$$

and there exist positive constants $C_{1}$ and $C_{2}$ such that

$$
\begin{aligned}
0 \leq a(\nu, T) & \leq C_{1}, \forall(\nu, T) \in(0, \infty) \times[m, M] \\
\left|a\left(\nu, T_{1}\right)-a\left(\nu, T_{2}\right)\right| & \leq C_{2}\left|T_{1}-T_{2}\right|, \forall\left(\nu, T_{i}\right) \in(0, \infty) \times[m, M] .
\end{aligned}
$$


Let us set

$$
\begin{aligned}
X & =\mathrm{L}^{1}(\Omega) \times \mathrm{L}^{1}\left((0, \infty) \times \Omega \times S^{2}\right), \\
\|(T, i)\|_{X} & =\|T\|_{\mathrm{L}^{1}(\Omega)}+\|i\|_{\mathrm{L}^{1}\left((0, \infty) \times \Omega \times S^{2}\right)}, \\
f & =\frac{F}{\rho c_{v}}, \text { and } \\
\|f\|_{\infty} & =\|f\|_{\mathrm{L}^{\infty}(\Omega \times[0, \tau])} .
\end{aligned}
$$

Then we have the following theorem, where the concept of solution is weak in the sense of the nonlinear semigroup theory (mild solution in [1] and [7], $\mathrm{C}^{0}$-solution in [18]).

Theorem 3.1 Under the previous hypotheses, the convection-conductionradiation problem has a unique solution $\{T, i\} \in \mathrm{C}([0, \tau] ; X)$ satisfying

$$
m \leq T \leq M \quad \text { and } \quad i_{b}(\nu, m) \leq i \leq i_{b}(\nu, M)
$$

provided that

- $m+\tau\|f\|_{\infty} \leq T_{0} \leq M-\tau\|f\|_{\infty}$ a.e. in $\Omega$,

- $m+\tau\|f\|_{\infty} \leq \bar{g} \leq M-\tau\|f\|_{\infty}$ a.e. on $\Gamma_{1}$,

- $i_{b}\left(\nu, m+\tau\|f\|_{\infty}\right) \leq i_{0} \leq i_{b}\left(\nu, M-\tau\|f\|_{\infty}\right)$ a.e. in $(0, \infty) \times \Omega \times S^{2}$,

- $i_{b}\left(\nu, m+\tau\|f\|_{\infty}\right) \leq \bar{h} \leq i_{b}\left(\nu, M-\tau\|f\|_{\infty}\right)$ a.e. in $(0, \infty) \times D_{\text {in }}$.

Remark 3.1 The assumption div $\mathbf{v}=0$ in Theorem 3.1 can be weakened in the hypothesis div $\mathbf{v} \leq 0$, although it is not clear the physical meaning of $\operatorname{div} \mathbf{v}<0$ in the energy equation (2.3).

Remark 3.2 Theorem 3.1 is valid in the particular case of the conductionradiation problem, which corresponds to the choice $\mathbf{v} \equiv \mathbf{0}$, for the problem with no internal heating $(f \equiv 0)$, and for the combination of both of them.

Remark 3.3 When $f \equiv 0$, the solution exists in the time interval $[0, \infty)$.

Remark 3.4 When the internal heat source $F$ is nonnegative ( $f$ nonnegative) then the same result holds if we replace $m+\tau\|f\|_{\infty}$ by $m$. This fact has the physical explanation that the temperature can only increase with time, and not decrease, due to the effect of a nonnegative heat source. 


\section{Application of the accretive operators theory}

The basic result that we use is a perturbed version of Crandall-Liggett generation theorem [1], [7], [18], which states that, if $X$ is a Banach space, the initial value problem $(d u / d t)+A u+B u=\bar{f}, u(0)=u_{0}$, has a unique (mild) solution provided that $A: D(A) \subset X \rightarrow X$ is m-accretive, $B: X \rightarrow X$ is Lipschitz, $\bar{f} \in \mathrm{L}^{1}(0, \tau ; X)$, and $u_{0} \in \overline{D(A)}$.

We can write the problem (2.8)-(2.11) in the abstract form

$$
\frac{d u}{d t}+A u+B u=\bar{f}, u(0)=\left\{T_{0}, i_{0}\right\}
$$

where $u=\{T, i\}, \bar{f}=\{f, 0\}$, and the operators $A$ and $B$ are defined as follows:

$$
\begin{aligned}
& A u=\left\{\mathbf{v} \cdot \nabla T-\frac{\bar{k}}{\rho c_{v}} \Delta T, c \boldsymbol{\omega} \cdot \nabla_{\mathbf{x}} i-c L(i)\right\} . \\
& B u=\left\{-\frac{1}{\rho c_{v}} \int_{0}^{\infty} \int_{S^{2}} q(u) d \nu d \boldsymbol{\omega}, c q(u)\right\}
\end{aligned}
$$

with

$$
q(u)(\nu, \mathbf{x}, \boldsymbol{\omega})=a(\nu, T(\mathbf{x}))\left[i(\nu, \mathbf{x}, \boldsymbol{\omega})-i_{b}(\nu, T(\mathbf{x}))\right]
$$

and

$$
X=\mathrm{L}^{1}(\Omega) \times \mathrm{L}^{1}\left((0, \infty) \times \Omega \times S^{2}\right) .
$$

The domain of $A$ is

$$
\begin{gathered}
D(A)=\left\{u=\{T, i\} \in X: \boldsymbol{\omega} \cdot \nabla_{\mathbf{x}} i \in \mathrm{L}^{1}\left((0, \infty) \times \Omega \times S^{2}\right),\right. \\
\left.T \in \mathrm{W}^{1,1}(\Omega), \Delta T \in \mathrm{L}^{1}(\Omega), \quad i \text { and } T \text { satisfy the b.c. }\right\} .
\end{gathered}
$$

The proof of the following lemma is given in the appendix.

Lemma 4.1 $D(A)$ is dense in $X$.

Lemma 4.1 guarantees that the initial condition $\left\{T_{0}, i_{0}\right\}$ can be chosen an arbitrary element of $X$.

When $\Gamma \backslash \Gamma_{1} \neq \emptyset$, we have to give sense to the b.c.

$$
\nabla T \cdot \mathbf{n}=0 \quad \text { on } \Gamma \backslash \Gamma_{1}
$$

This is done in the following definition. 
Definition 4.1 Given $T \in \mathrm{W}^{1,1}(\Omega)$ such that $\Delta T \in \mathrm{L}^{1}(\Omega)$, we say that $T$ satisfies the b.c. (4.7) if

$$
\int_{\Omega} \Delta T \varphi d \mathbf{x}=-\int_{\Omega} \nabla T \cdot \nabla \varphi d \mathbf{x}
$$

for all $\varphi \in \mathrm{H}^{1}(\Omega) \cap \mathrm{W}^{1, \infty}(\Omega)$ such that $\varphi_{\mid \Gamma_{1}}=0$.

Remark 4.1 Note that, if $T \in \mathrm{W}^{2,1}(\Omega)$, (4.8) implies

$$
\int_{\Gamma \backslash \Gamma_{1}}(\nabla T \cdot \mathbf{n}) \varphi d \gamma=0
$$

for all $\varphi \in \mathrm{H}^{1}(\Omega) \cap \mathrm{W}^{1, \infty}(\Omega)$ such that $\varphi_{\mid \Gamma_{1}}=0$.

The operator $B$ is not well defined from $X$ into $X$; instead, we consider a "truncation" $B_{m}^{M}$ which is well defined and Lipschitz, if the hypotheses described in Section 3 are fulfilled. Before defining $B_{m}^{M}$, we need some other definitions. Recall that $m$ and $M$ are two real numbers such that $0<m<M$. In the following definition $\zeta$ may be $a$ or $i_{b}$ :

$$
\Theta_{m}^{M}(\zeta)(\nu, T)=\left\{\begin{array}{lll}
0 & \text { if } & T<0 \\
(\zeta(\nu, m) / m) T & \text { if } & 0 \leq T<m \\
\zeta(\nu, T) & \text { if } & m \leq T \leq M \\
\zeta(\nu, M) & \text { if } & T>M .
\end{array}\right.
$$

Remark 4.2 Since $m>0, \Theta_{m}^{M}(a)$ maintains us away from the singularity of the absorption coefficient a at $T=0$ [10].

Let us define also

$$
\varphi^{M}(i)(\nu, \mathbf{x}, \boldsymbol{\omega})= \begin{cases}0 & \text { if } i(\nu, \mathbf{x}, \boldsymbol{\omega})<0 \\ i(\nu, \mathbf{x}, \boldsymbol{\omega}) & \text { if } 0 \leq i(\nu, \mathbf{x}, \boldsymbol{\omega}) \leq i_{b}(\nu, M) \\ i_{b}(\nu, M) & \text { if } i(\nu, \mathbf{x}, \boldsymbol{\omega})>i_{b}(\nu, M)\end{cases}
$$

Now $B_{m}^{M}$ is given, for all $u \in X$, by

$$
B_{m}^{M} u=\left\{-\frac{1}{\rho c_{v}} \int_{0}^{\infty} \int_{S^{2}} q_{m}^{M}(u) d \boldsymbol{\omega} d \nu, c q_{m}^{M}(u)\right\},
$$

where

(4.12) $q_{m}^{M}(u)(\nu, \mathbf{x}, \boldsymbol{\omega})=\Theta_{m}^{M}(a)(\nu, T(\mathbf{x}))\left[\varphi^{M}(i)(\nu, \mathbf{x}, \boldsymbol{\omega})-\Theta_{m}^{M}\left(i_{b}\right)(\nu, T(\mathbf{x}))\right]$.

Clearly, it results $B_{m}^{M} u=B u$ whenever

$$
m \leq T \leq M \quad \text { and } \quad 0 \leq i \leq i_{b}(\nu, M)
$$


Let us call the truncated problem the one obtained by replacing in (4.1) $B$ by $B_{m}^{M}$. The plan of the paper is the following:

(1) Prove that $A$ is m-accretive (that is, accretive and $R(I+\lambda A)=X$ for $\lambda>0$, where $R(I+\lambda A)$ is the range of $I+\lambda A)$.

(2) Prove that $B_{m}^{M}$ is Lipschitz.

(3) Give conditions on the initial and boundary data in order that the unique solution of the truncated problem satisfies $m \leq T \leq M$ and $i_{b}(\nu, m) \leq i \leq i_{b}(\nu, M)$, which implies that it is also solution of (4.1).

Once we know the results in [16], basically we have to deal only with the convective and conductive terms (see also [14]).

In what concerns the proof of accretivity, some integral estimates are needed, the proofs of which are given in this paper; the key for proving that $R(I+\lambda A)=X$ for $\lambda>0$ is the existence (uniqueness is always guaranteed by the accretivity) for the elliptic linear problem

$$
T+\lambda \mathbf{v} \cdot \nabla T-\lambda C \Delta T=\hat{T},
$$

with mixed $\left(T=\bar{g}\right.$ on $\Gamma_{1}, \nabla T \cdot \mathbf{n}=0$ on $\left.\Gamma \backslash \Gamma_{1}\right)$ b.c., where $C$ is a positive constant and $\hat{T} \in \mathrm{L}^{1}(\Omega)$; we have proved in [15] that this result holds. Item (2) is known from [14]. To solve item (3) we adapt the techniques of [16] and use analogous arguments to those used to prove accretivity.

\section{1. m-accretivity of $A$}

Recall the definition of the sign function:

$$
\operatorname{sgn}(t)=\left\{\begin{array}{cll}
-1 & \text { if } & t<0 \\
0 & \text { if } & t=0 \\
1 & \text { if } & t>0
\end{array}\right.
$$

\subsubsection{Accretivity}

The operator $A$ is accretive if, and only if, the following inequality is satisfied for all $\{T, i\}$ and $\{\hat{T}, \hat{\imath}\}$ in $D(A)$ :

$$
\begin{gathered}
\int_{\Omega}\left[\mathbf{v} \cdot \nabla(T-\hat{T})-\frac{\bar{k}}{\rho c_{v}} \Delta(T-\hat{T})\right] \operatorname{sgn}(T-\hat{T}) d \mathbf{x}+ \\
\int_{\{T=\hat{T}\}}\left|\mathbf{v} \cdot \nabla(T-\hat{T})-\frac{\bar{k}}{\rho c_{v}} \Delta(T-\hat{T})\right| d \mathbf{x}+ \\
\int_{0}^{\infty} \int_{\Omega} \int_{S^{2}}\left[c \boldsymbol{\omega} \cdot \nabla_{\mathbf{x}}(i-\hat{\imath})-c L(i-\hat{\imath})\right] \operatorname{sgn}(i-\hat{\imath}) d \boldsymbol{\omega} d \mathbf{x} d \nu+ \\
\int_{\{i=\hat{\imath}\}}\left|c \boldsymbol{\omega} \cdot \nabla_{\mathbf{x}}(i-\hat{\imath})-c L(i-\hat{\imath})\right| d \boldsymbol{\omega} d \mathbf{x} d \nu \geq 0 .
\end{gathered}
$$


In fact, we show that every term in the sum in nonnegative. Obviously, the second and fourth integrals are nonnegative. Moreover, we know from [16] that the third one is nonnegative. To prove that the first one is nonnegative note that, since $\operatorname{div} \mathbf{v}=0$,

$$
\begin{aligned}
\int_{\Omega} \mathbf{v} & \cdot \nabla(T-\hat{T}) \operatorname{sgn}(T-\hat{T}) d \mathbf{x}=\int_{\Omega} \mathbf{v} \cdot \nabla(|T-\hat{T}|) d \mathbf{x} \\
= & \int_{\Gamma}|T-\hat{T}| \mathbf{v} \cdot \mathbf{n} d \gamma=\int_{\Gamma \backslash \Gamma_{1}}|T-\hat{T}| \mathbf{v} \cdot \mathbf{n} d \gamma \geq 0
\end{aligned}
$$

where we have used the facts that $T=\hat{T}=\bar{g}$ on $\Gamma_{1}$ and that $\Gamma_{\mathbf{v}}^{-} \subseteq \Gamma_{1}$.

The proof of the accretivity of $A$ ends by proving that

$$
-\int_{\Omega} \Delta(T-\hat{T}) \operatorname{sgn}(T-\hat{T}) d \mathbf{x} \geq 0
$$

which is a consequence of the following lemma, the proof of which is given in the appendix.

Lemma 4.2 Let us take $\beta \in \mathrm{C}^{1}(\mathbb{R}) \cap \mathrm{L}^{\infty}(\mathbb{R})$ such that $\beta(0)=0$ and $\beta^{\prime} \geq 0$, and let $\Gamma_{1}$ be an arbitrary measurable subset of $\Gamma$. Then, for all $T \in \mathrm{W}^{1,1}(\Omega)$ such that $\Delta T \in \mathrm{L}^{1}(\Omega), T=0$ on $\Gamma_{1}$ and $\nabla T \cdot \mathbf{n}=0$ on $\Gamma \backslash \Gamma_{1}$ (in the sense of Definition 4.1),

$$
-\int_{\Omega} \Delta T \beta(T) d \mathbf{x} \geq 0
$$

Now (4.16) holds in virtue of the following corollary.

Corollary 4.1 Let $\Gamma_{1}$ and $T$ be as in the Lemma 4.2. Then

$$
-\int_{\Omega} \Delta T \operatorname{sgn}(T) d \mathbf{x} \geq 0
$$

Proof. Let us consider $\left\{\beta_{n}\right\} \subset \mathrm{C}^{1}(\mathbb{R}) \cap \mathrm{L}^{\infty}(\mathbb{R})$ such that $\left\{\beta_{n}\right\} \rightarrow$ sgn pointwise, $\beta_{n}(0)=0, \beta_{n}^{\prime} \geq 0$ and $\left\|\beta_{n}\right\|_{L^{\infty}(\mathbb{R})} \leq C$ for all $n$, with $C$ independent of $n$. According to Lemma 4.2,

$$
-\int_{\Omega} \Delta T \beta_{n}(T) d \mathbf{x} \geq 0
$$

and the result follows by taking the limit as $n \rightarrow \infty$. 


\subsection{2. m-accretivity}

The m-accretivity means, first, accretivity, and, second, that if

$$
\hat{u}=\{\hat{T}, \hat{\imath}\} \in X \quad \text { and } \quad \lambda>0,
$$

then the problem $u+\lambda A u=\hat{u}$ has some solution $u \in D(A)$ (this solution is necessarily unique in virtue of accretivity). Since the operator $A$ is m-accretive when $\mathbf{v} \equiv \mathbf{0}$ and $\bar{k}=0$ [16], it suffices to prove that, for $\hat{T}$ given in $\mathrm{L}^{1}(\Omega)$ and $\lambda>0$, the problem

$$
T+\lambda \mathbf{v} \cdot \nabla T-\lambda \frac{\bar{k}}{\rho c_{v}} \Delta T=\hat{T}
$$

has a unique distributional solution $T \in \mathrm{W}^{1,1}(\Omega)$ with the mixed b.c.

$$
\begin{aligned}
T=\bar{g} & \text { on } \Gamma_{1}, \\
\nabla T \cdot \mathbf{n}=0 & \text { on } \Gamma \backslash \Gamma_{1},
\end{aligned}
$$

where $\Gamma_{1}$ is a measurable subset of $\Gamma$. This is indeed the case, and the reader can find the proof in [15, Theorem 2.3].

\subsection{Boundedness of the solution of the truncated problem}

The functions $\mathrm{s}_{ \pm}: \mathbb{R} \rightarrow \mathbb{R}$ are defined by:

$$
\mathrm{s}_{+}(t)=\left\{\begin{array}{ll}
1 & \text { if } t>0 \\
0 & \text { if } t \leq 0,
\end{array} \quad \mathrm{~s}_{-}(t)=\left\{\begin{array}{cl}
-1 & \text { if } t<0 \\
0 & \text { if } t \geq 0 .
\end{array}\right.\right.
$$

If $H$ is a real function, set $H_{ \pm}=H \cdot \mathrm{s}_{ \pm}(H)$.

We assume that the hypotheses described in Section 3 are satisfied. Recall that $f=F /\left(\rho c_{v}\right)$ and $\bar{f}=\{f, 0\}$. Let $u=\{T, i\} \in \mathrm{C}^{0}([0, \tau] ; X)$ be the unique (mild) solution of the truncated problem.

We can choose [1], [13, Lemma 2.2.2] a family of partitions of $[0, \tau]$

$$
0=t_{0}^{n}<\cdots<t_{P(n)}^{n}=\tau \quad(n \in \mathbb{N})
$$

and a family of finite sequences in $X$

$$
\bar{f}_{1}^{n}=\left\{f_{1}^{n}, 0\right\}, \ldots, \bar{f}_{P(n)}^{n}=\left\{f_{P(n)}^{n}, 0\right\}
$$

such that

$$
\lim _{n \rightarrow \infty} \max _{1 \leq j \leq P(n)} \lambda_{j}^{n}=\lim _{n \rightarrow \infty}\left\|\bar{f}-\bar{f}_{n}\right\|_{\mathrm{L}^{1}(0, \tau ; X)}=0,
$$

where $\lambda_{j}^{n}=t_{j}^{n}-t_{j-1}^{n}$ and $\bar{f}_{n}(t)=\bar{f}_{j}^{n} \forall t \in\left(t_{j-1}^{n}, t_{j}^{n}\right]$, for $j=1, \ldots, P(n)$. 
Set $u_{0}^{n}=\left\{T_{0}^{n}, i_{0}^{n}\right\}=u_{0}=\left\{T_{0}, i_{0}\right\} \forall n \in \mathbb{N}$, and let $u_{j}^{n}=\left\{T_{j}^{n}, i_{j}^{n}\right\}$ be the unique solution of

$$
\frac{u_{j}^{n}-u_{j-1}^{n}}{\lambda_{j}^{n}}+A u_{j}^{n}+B_{m}^{M} u_{j}^{n}=\bar{f}_{j}^{n},
$$

for $j=1, \ldots, P(n)$.

Then $u$ is the uniform limit on $[0, \tau]$ of the sequence $\left\{u_{n}\right\}$, piecewise defined as follows:

$$
\begin{aligned}
& u_{n}(0)=u_{0}, \\
& u_{n}(t)=u_{j}^{n} \quad \text { if } \quad t \in\left(t_{j-1}^{n}, t_{j}^{n}\right] .
\end{aligned}
$$

Remark 4.3 Since $A$ is $m$-accretive and $B_{m}^{M}$ is Lipschitz with domain $X$, the problem (4.28) has a unique solution in $D(A)$ for small values of $\lambda_{j}^{n}$, to be precise for $0<\lambda_{j}^{n}<1 / M^{\star}$, where $M^{\star}$ is a Lipschitz constant for $B_{m}^{M}$. See [14, Theorem 2.3]; a proof can be done by using the Banach fixed point theorem.

At this point it must be clear that it results

$$
m \leq T \leq M \quad \text { and } \quad i_{b}(\nu, m) \leq i \leq i_{b}(\nu, M)
$$

if, for every $n \in \mathbb{N}$,

$$
m \leq T_{j}^{n}(\mathbf{x}) \leq M \quad \text { and } \quad i_{b}(\nu, m) \leq i_{j}^{n}(\nu, \mathbf{x}, \boldsymbol{\omega}) \leq i_{b}(\nu, M)
$$

$\forall j \in\{0,1, \ldots, P(n)\}$. We need the following lemma to obtain bounds for the stationary problem (4.28).

Lemma 4.3 Let us take: $f^{\star} \in \mathrm{L}^{1}(\Omega), M^{\star}$ a Lipschitz constant for $B_{m}^{M}$, $\lambda \in \mathbb{R}$ such that $0<\lambda<1 / M^{\star}$, and $\hat{u}=\{\hat{T}, \hat{\imath}\} \in X$. Let $u=\{T, i\} \in D(A)$ be the unique solution of

$$
u+\lambda\left(A u+B_{m}^{M} u\right)=\hat{u}+\left\{\lambda f^{\star}, 0\right\} .
$$

If $N^{\prime}$ and $N$ are constants such that $m \leq N^{\prime} \leq N \leq M$, then

$$
\begin{aligned}
N^{\prime} & \leq T \leq N \text { a.e. in } \Omega, \\
i_{b}\left(\nu, N^{\prime}\right) & \leq i \leq i_{b}(\nu, N) \text { a.e. in }(0, \infty) \times \Omega \times S^{2}
\end{aligned}
$$

provided that

- $N^{\prime} \leq \hat{T}+\lambda f^{\star} \leq N$ a.e. in $\Omega$,

- $N^{\prime} \leq \bar{g} \leq N$ a.e. on $\Gamma_{1}$,

- $i_{b}\left(\nu, N^{\prime}\right) \leq \hat{\imath} \leq i_{b}(\nu, N)$ a.e. in $(0, \infty) \times \Omega \times S^{2}$, and

- $i_{b}\left(\nu, N^{\prime}\right) \leq \bar{h} \leq i_{b}(\nu, N)$ a.e. in $(0, \infty) \times D_{\text {in }}$. 
Proof. We prove $T \leq N$ and $i \leq i_{b}(N)$, and what is left can be proved analogously. In this proof we use the shortened notation $i_{b}(N)=i_{b}(\nu, N)$. From the equation $u+\lambda\left(A u+B_{m}^{M} u\right)=\hat{u}+\left\{\lambda f^{\star}, 0\right\}$ it follows

$$
\rho c_{v}\left\|(T-N)_{+}\right\|_{\mathrm{L}^{1}(\Omega)}+\frac{1}{c}\left\|\left(i-i_{b}(N)\right)_{+}\right\|_{\mathrm{L}^{1}\left((0, \infty) \times \Omega \times S^{2}\right)}+
$$

$+\lambda \int_{\Omega} \rho c_{v} \mathbf{v} \cdot \nabla(T-N) \mathrm{s}_{+}(T-N) d \mathbf{x}$

$+\lambda \int_{0}^{\infty} \int_{\Omega} \int_{S^{2}} \boldsymbol{\omega} \cdot \nabla_{\mathbf{x}} i \mathrm{~s}_{+}\left(i-i_{b}(N)\right) d \boldsymbol{\omega} d \mathbf{x} d \nu$

$+\lambda \int_{0}^{\infty} \int_{\Omega} \int_{S^{2}} \Theta_{m}^{M}(a)\left[\Theta_{m}^{M}\left(i_{b}\right)-i_{b}(N)\right]\left[\mathrm{s}_{+}(T-N)-\mathrm{s}_{+}\left(i-i_{b}(N)\right)\right] d \boldsymbol{\omega} d \mathbf{x} d \nu$

$+\lambda \int_{0}^{\infty} \int_{\Omega} \int_{S^{2}} \Theta_{m}^{M}(a)\left[\varphi^{M}(i)-i_{b}(N)\right]\left[\mathrm{s}_{+}\left(i-i_{b}(N)\right)-\mathrm{s}_{+}(T-N)\right] d \boldsymbol{\omega} d \mathbf{x} d \nu$

$=\int_{\Omega} \rho c_{v}\left(\hat{T}+\lambda f^{*}-N\right) \mathrm{s}_{+}(T-M) d \mathbf{x}$

$$
+\frac{1}{c} \int_{0}^{\infty} \int_{\Omega} \int_{S^{2}}\left(\hat{\imath}-i_{b}(N)\right) \mathrm{s}_{+}\left(i-i_{b}(N)\right) d \boldsymbol{\omega} d \mathbf{x} d \nu
$$

$+\lambda \int_{\Omega} \bar{k} \Delta T \mathrm{~s}_{+}(T-N) d \mathbf{x}+\lambda \int_{0}^{\infty} \int_{\Omega} \int_{S^{2}} L(i) \mathrm{s}_{+}\left(i-i_{b}(N)\right) d \boldsymbol{\omega} d \mathbf{x} d \nu$.

The idea is to prove that all the terms in the left-hand side are nonnegative and all the terms in the right-hand side are nonpositive, since this implies

$$
\rho c_{v}\left\|(T-N)_{+}\right\|+(1 / c)\left\|\left(i-i_{b}(N)\right)_{+}\right\| \leq 0,
$$

as desired. Taking into account that the case with $\mathbf{v} \equiv \mathbf{0}$ and $\bar{k}=0$ has already be studied [16], [13] and [14], it suffices to prove

$$
\int_{\Omega} \mathbf{v} \cdot \nabla(T-N) \mathrm{s}_{+}(T-N) d \mathbf{x} \geq 0
$$

and

$$
-\int_{\Omega} \Delta T \mathrm{~s}_{+}(T-N) d \mathbf{x} \geq 0
$$

The proof becomes now similar to that of accretivity: since div $\mathbf{v}=0$,

$$
\begin{aligned}
\int_{\Omega} \mathbf{v} \cdot \nabla(T-N) \mathrm{s}_{+}(T-N) d \mathbf{x} & =\int_{\Gamma}(T-N)_{+} \mathbf{v} \cdot \mathbf{n} d \gamma \\
& =\int_{\Gamma \backslash \Gamma_{1}}(T-N)_{+} \mathbf{v} \cdot \mathbf{n} d \gamma \geq 0
\end{aligned}
$$

where we have taken into account that $T=\bar{g} \leq N$ on $\Gamma_{1}$ and that $\Gamma_{\mathbf{v}}^{-} \subseteq \Gamma_{1}$. 
The inequality (4.38) is a consequence of the following lemma, the proof of which is given in the appendix.

Lemma 4.4 Let $\Gamma_{1}$ be an arbitrary measurable subset of $\Gamma$, and let us suppose that $g$ is such that $g=\psi_{\mid \Gamma_{1}}$ for certain $\psi \in \mathcal{D}(\bar{\Omega})$ and that $g \leq 0$ on $\Gamma_{1}$. Let us take $\beta \in \mathrm{C}^{1}(\mathbb{R}) \cap \mathrm{L}^{\infty}(\mathbb{R})$ such that $\beta(s)=0 \forall s \leq 0$ and $\beta^{\prime} \geq 0$. Then, for all $T \in \mathrm{W}^{1,1}(\Omega)$ such that $\Delta T \in \mathrm{L}^{1}(\Omega), T=g$ on $\Gamma_{1}$ and $\nabla T \cdot \mathbf{n}=0$ on $\Gamma \backslash \Gamma_{1}$ (in the sense of Definition 4.1),

$$
-\int_{\Omega} \Delta T \beta(T) d \mathbf{x} \geq 0
$$

Now (4.38) holds in virtue of the following corollary, since

$$
\Delta T=\Delta(T-N)
$$

and $T-N=\bar{g}-N \leq 0$ on $\Gamma_{1}$.

Corollary 4.2 Let $\Gamma_{1}, g$ and $T$ be as in the Lemma 4.4. Then

$$
-\int_{\Omega} \Delta T \mathrm{~s}_{+}(T) d \mathbf{x} \geq 0 .
$$

The proof of the Corollary 4.2 follows closely the arguments used to prove Corollary 4.1. This ends the proof of Lemma 4.3.

Finally, the following theorem gives Theorem 3.1 simply by taking $N^{\prime}=m$ and $N=M$. Note that a new restriction arises for $m$ and $M$, since they must satisfy $m+2 \tau\|f\|_{\infty} \leq M$. Recall that $\|f\|_{\infty}=\|f\|_{\mathrm{L}^{\infty}(\Omega \times[0, \tau])}$.

Theorem 4.1 Let $N^{\prime}$ and $N$ be constants such that

$$
m \leq N^{\prime}, \quad N^{\prime}+\tau\|f\|_{\infty} \leq N-\tau\|f\|_{\infty}, \quad \text { and } \quad N \leq M .
$$

Let $u=\{T, i\}$ be the solution of the truncated problem. Then u satisfies

$$
\begin{aligned}
N^{\prime} & \leq T \leq N \quad \text { a.e. in } \Omega, \\
i_{b}\left(\nu, N^{\prime}\right) & \leq i \leq i_{b}(\nu, N) \text { a.e. in }(0, \infty) \times \Omega \times S^{2}
\end{aligned}
$$

for all $t \in[0, \tau]$, provided that

- $N^{\prime}+\tau\|f\|_{\infty} \leq T_{0} \leq N-\tau\|f\|_{\infty}$ a.e. in $\Omega$,

- $N^{\prime}+\tau\|f\|_{\infty} \leq \bar{g} \leq N-\tau\|f\|_{\infty}$ a.e. on $\Gamma_{1}$,

- $i_{b}\left(\nu, N^{\prime}+\tau\|f\|_{\infty}\right) \leq i_{0} \leq i_{b}\left(\nu, N-\tau\|f\|_{\infty}\right)$ a.e. in $(0, \infty) \times \Omega \times S^{2}$,

- $i_{b}\left(\nu, N^{\prime}+\tau\|f\|_{\infty}\right) \leq \bar{h} \leq i_{b}\left(\nu, N-\tau\|f\|_{\infty}\right)$ a.e. in $(0, \infty) \times D_{\text {in }}$. 
Proof. In this proof $\|f\|$ is $\|f\|_{\infty}$. By previous discussion it suffices to prove (4.32) replacing $m$ and $M$ by $N^{\prime}$ and $N$, respectively. Note that

$$
N^{\prime}+\tau\|f\| \leq T_{0}^{n}=T_{0} \leq N-\tau\|f\|
$$

and

$$
i_{b}\left(\nu, N^{\prime}+\tau\|f\|\right) \leq i_{0}^{n}=i_{0} \leq i_{b}(\nu, N-\tau\|f\|) .
$$

By succesive application of Lemma 4.3 for $j=1, \ldots, P(n)$ we obtain

$$
\begin{aligned}
& N^{\prime}+\left(\tau-\sum_{\alpha=1}^{j} \lambda_{\alpha}^{n}\right)\|f\| \leq T_{j}^{n} \leq N-\left(\tau-\sum_{\alpha=1}^{j} \lambda_{\alpha}^{n}\right)\|f\|, \\
& i_{b}\left(\nu, N^{\prime}+\left(\tau-\sum_{\alpha=1}^{j} \lambda_{\alpha}^{n}\right)\|f\|\right) \leq i_{j}^{n} \leq i_{b}\left(\nu, N-\left(\tau-\sum_{\alpha=1}^{j} \lambda_{\alpha}^{n}\right)\|f\|\right),
\end{aligned}
$$

which proves $(4.32)$, since $i_{b}(\nu, \cdot)$ is increasing and $\sum_{\alpha=1}^{P(n)} \lambda_{\alpha}^{n}=\tau$.

\section{Conclusions}

We have proved solvability of the transient radiative transfer equations for a participating and incompressible gas in the presence of conduction and the possible presence of convection. There are some clear limits in this work, perhaps the more important one is that the velocity field and the boundary data must be stationary, due (in principle) only to technical reasons, since otherwise our operator $A$ would depend on time, making more difficult the treatment of the problem.

\section{Appendix}

In this appendix we give the proofs of Lemmas 4.1, 4.2 and 4.4. Let us recall that $\Omega$ is a bounded, open and connected subset of $\mathbb{R}^{3}$, with $\mathrm{C}^{\infty}$-boundary $\Gamma$.

Proof of Lemma 4.1. Here $\Gamma_{1}$ is an arbitrary measurable subset of $\Gamma$. Note that, once we know [14, Corollary 1], it suffices to prove that, given $f \in \mathrm{L}^{1}(\Omega)$, there exists a sequence $\left\{T_{n}\right\} \subset \mathrm{W}^{1,1}(\Omega)$ converging to $f$ in $\mathrm{L}^{1}(\Omega)$ such that $\Delta T_{n} \in \mathrm{L}^{1}(\Omega), T_{n}=\bar{g}$ on $\Gamma_{1}$, and $\nabla T_{n} \cdot \mathbf{n}=0$ on $\Gamma \backslash \Gamma_{1}$.

Let $u$ be the unique solution of the following elliptic problem:

$$
\left\{\begin{array}{l}
u-\Delta u=0, \\
u=\bar{g} \text { on } \Gamma_{1}, \\
\nabla u \cdot \mathbf{n}=0 \text { on } \Gamma \backslash \Gamma_{1} .
\end{array}\right.
$$


Since $f-u \in \mathrm{L}^{1}(\Omega)$ and $\mathcal{D}(\Omega)$ is dense in $\mathrm{L}^{1}(\Omega)$, there exists a sequence

$$
\left\{\varphi_{n}\right\} \subset \mathcal{D}(\Omega) \text { such that }\left\{\varphi_{n}\right\} \rightarrow f-u \text { in } \mathrm{L}^{1}(\Omega) .
$$

Now

$$
T_{n}=\varphi_{n}+u
$$

defines the desired sequence converging to $f$.

To prove Lemma 4.2 and Lemma 4.4 we use the following result, which is a direct consequence of [15, Theorem 6.1, Remark 6.1].

Lemma 6.1 Let $\Gamma_{1}$ be an arbitrary measurable subset of $\Gamma$. Given $\hat{T} \in$ $\mathrm{L}^{1}(\Omega)$, the problem

$$
\left\{\begin{array}{rlrl}
T-\Delta T & =\hat{T}, & \\
T=0 & & \text { on } \Gamma_{1}, \\
\nabla T \cdot \mathbf{n} & =0 & & \text { on } \Gamma \backslash \Gamma_{1},
\end{array}\right.
$$

has a unique distributional solution, i.e., there exists a unique $T \in \mathrm{W}^{1,1}(\Omega)$ such that $T_{\mid \Gamma_{1}}=0$ and

$$
\int_{\Omega} T \varphi d \mathbf{x}+\int_{\Omega} \nabla T \cdot \nabla \varphi d \mathbf{x}=\int_{\Omega} \hat{T} \varphi d \mathbf{x}
$$

for every test function $\varphi \in \mathrm{W}^{1, \infty}(\Omega)$ satisfying $\varphi_{\mid \Gamma_{1}}=0$. Moreover, this solution satisfies

$$
\|T\|_{\mathrm{L}^{1}(\Omega)} \leq c\|\hat{T}\|_{\mathrm{L}^{1}(\Omega)},
$$

where $c$ is a constant which depends only on $|\Omega|$.

The following result contains as particular cases both Lemma 4.2 and Lemma 4.4.

Lemma 6.2 Let $\Gamma_{1}$ be an arbitrary measurable subset of $\Gamma$, and let us suppose that $g$ is such that $g=\psi_{\mid \Gamma_{1}}$ for certain $\psi \in \mathcal{D}(\bar{\Omega})$. Let us take $\beta \in \mathrm{C}^{1}(\mathbb{R}) \cap \mathrm{L}^{\infty}(\mathbb{R})$ such that $\beta \circ g=0$ on $\Gamma_{1}$ and $\beta^{\prime} \geq 0$. Then, for all $T \in \mathrm{W}^{1,1}(\Omega)$ such that $\Delta T \in \mathrm{L}^{1}(\Omega), T=g$ on $\Gamma_{1}$ and $\nabla T \cdot \mathbf{n}=0$ on $\Gamma \backslash \Gamma_{1}$ (in the sense of Definition 4.1),

$$
-\int_{\Omega} \Delta T \beta(T) d \mathbf{x} \geq 0
$$


Proof. Set $f=T-\Delta T$. Note that $f \in \mathrm{L}^{1}(\Omega)$, and thus there exists a sequence

$$
\left\{f_{n}\right\} \subset \mathcal{D}(\Omega) \text { such that }\left\{f_{n}\right\} \rightarrow f \quad \text { in } \mathrm{L}^{1}(\Omega) .
$$

Denote by $T_{n}$ the unique solution of the following problem:

$$
\left\{\begin{array}{l}
T_{n}-\Delta T_{n}=f_{n}, \\
T_{n}=g \text { on } \Gamma_{1}, \\
\nabla T_{n} \cdot \mathbf{n}=0 \text { on } \Gamma \backslash \Gamma_{1} .
\end{array}\right.
$$

Then $T_{n}$ is regular, and we can integrate by parts to obtain

$$
-\int_{\Omega} \Delta T_{n} \beta\left(T_{n}\right) d \mathbf{x}=\int_{\Omega}\left|\nabla T_{n}\right|^{2} \beta^{\prime}\left(T_{n}\right) d \mathbf{x}-\int_{\Gamma} \beta\left(T_{n}\right) \nabla T_{n} \cdot \mathbf{n} d \gamma
$$

which proves that

$$
-\int_{\Omega} \Delta T_{n} \beta\left(T_{n}\right) d \mathbf{x} \geq 0
$$

since $\beta\left(T_{n}\right)=\beta(g)=0$ on $\Gamma_{1}$ and $\nabla T_{n} \cdot \mathbf{n}=0$ on $\Gamma \backslash \Gamma_{1}$. The proof ends by noting that, in virtue of Lemma 6.1,

$$
\left\|T-T_{n}\right\|_{\mathrm{L}^{1}(\Omega)} \leq c\left\|f-f_{n}\right\|_{\mathrm{L}^{1}(\Omega)},
$$

which implies

$$
\left\{T_{n}\right\} \rightarrow T \text { in } \mathrm{L}^{1}(\Omega)
$$

and consequently, at least for a certain subsequence $\left\{T_{n_{k}}\right\}$ of $\left\{T_{n}\right\}$ (which converges also a.e. to $T$ ),

$$
-\int_{\Omega} \Delta T \beta(T) d \mathbf{x}=-\lim _{k \rightarrow \infty} \int_{\Omega} \Delta T_{n_{k}} \beta\left(T_{n_{k}}\right) d \mathbf{x} \geq 0,
$$

as desired.

\section{References}

[1] Bénilan, Ph., Crandall, M. G. and Pazy, A.: Nonlinear Evolution Governed by Accretive Operators. Springer, to appear.

[2] Betta, M. F., Del Vecchio, T. and Posteraro, M. R.: Existence and regularity results for nonlinear degenerate elliptic equations with measure data. Ricerche Mat. 47 (1998), no. 2, 277-295. 
[3] Betta, M. F., Mercaldo, A., Murat, F. and Porzio, M. M.: Existence and uniqueness results for nonlinear elliptic problems with a lower order term and measure datum. C. R. Math. Acad. Sci. Paris 334 (2002), no. $9,757-762$.

[4] Betta, M.F., Mercaldo, A., Murat, F. and Porzio, M. M.: Existence of renormalized solutions to nonlinear elliptic equations with lowerorder terms and right-hand side a measure. J. Math. Pures Appl. (9) 82 (2003), no. 1, 90-124.

[5] Betta, M. F., Mercaldo, A., Murat, F. and Porzio, M. M.: Uniqueness of renormalized solutions to nonlinear elliptic equations with a lower order term and right hand side in $L^{1}(\Omega)$. ESAIM Control Optim. Calc. Var. 8 (2002) (Special volume: A tribute to Jacques-Louis Lions), 239-272.

[6] Candel, S.: Mécanique des fluides. Dunod, Paris, 1990.

[7] CRandall, M. G.: Nonlinear semigroups and evolution governed by accretive operators (1983). In Nonlinear Functional Analysis and Its Applications, 305-337, (F.E. Browder, ed.). Proc. Sympos. Pure Math. 45 (part 1). American Mathematical Society, Providence, RI, 1986.

[8] Del Vecchio, T.: Nonlinear elliptic equations with measure data. Potential Anal. 4 (1995), no. 2, 185-203.

[9] Del Vecchio, T. and Posteraro, M. R.: Existence and regularity results for nonlinear elliptic equations with measure data. Adv. Differential Equations 1 (1996), no. 5, 899-917.

[10] Golse, F. and Perthame, B.: Generalized solutions of the radiative transfer equations in a singular case. Comm. Math. Phys. 106 (1986), no. $2,211-239$.

[11] Kelley, C. T.: Existence and uniqueness of solutions of nonlinear systems of conductive-radiative heat transfer equations. Transport Theory Statist. Phys. 25 (1996), no. 2, 249-260.

[12] Laitinen, M. T. And Tilhonen, T.: Integro-differential equation modelling heat transfer in conducting, radiating and semitransparent materials. Math. Methods Appl. Sci. 21 (1998), no. 5, 375-392.

[13] López-Pouso, Ó.: Espacios de distribuciones, operadores acretivos y radiación térmica. Ph. D. Thesis. Publicaciones del Departamento de Matemática Aplicada 20, Universidad de Santiago de Compostela, 1999.

[14] López-Pouso, Ó.: Trace theorem and existence in radiation. Adv. Math. Sci. Appl. 10 (2000), no. 2, 757-773.

[15] López-Pouso, Ó. And Porzio, M. M.: Linear elliptic problems with mixed boundary conditions related to radiation heat transfer. Math. Models Methods Appl. Sci. 12 (2002), no. 4, 541-565.

[16] Mercier, B.: Application of accretive operators theory to the radiative transfer equations. SIAM J. Math. Anal. 18 (1987), no. 2, 393-408. 
[17] Modest, M. F.: Radiative Heat Transfer. McGraw-Hill Inc., New York, 1993.

[18] Showalter, R. E.: Monotone operators in Banach space and nonlinear partial differential equations. Mathematical Surveys and Monographs 49. American Mathematical Society, Providence, RI, 1997.

[19] Siegel, R.: Transient effects of radiative transfer in semitransparent materials. Internat. J. Engrg. Sci. 36 (1998), 1701-1739.

Recibido: 9 de mayo de 2002

Maria Michaela Porzio Facoltá di Scienze MM. FF. NN.

Universitá del Sannio via Port'Arsa 11 82100 Benevento, Italy porzio@unisannio.it

Óscar López-Pouso Departamento Matemática Aplicada Facultad de Matemáticas Universidad de Santiago de Compostela Campus Sur s/n 15782 Santiago de Compostela (A Coruña, Spain) oscarlp@usc.es 